Supporting Information for

\title{
Single Molecule Techniques Can Distinguish the Photophysical Processes Governing Metal-Enhanced Fluorescence
}

Gregory K. Hodgson, Nicholas P. Dogantzis and Stefania Impellizzeri*

Laboratory for Nanomaterials and Molecular Plasmonics, Department of Chemistry and

Biology, Ryerson University, 350 Victoria St., Toronto, ON, M5B 2K3, Canada.

Email: simpellizzeri@ryerson.ca

CONTENT

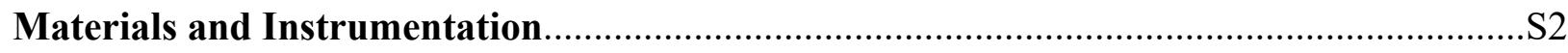

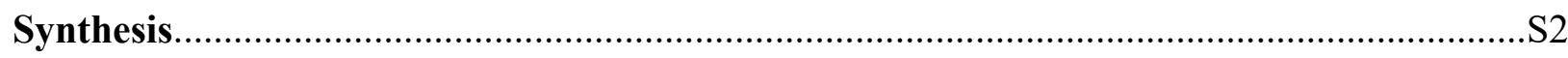

Fluorescence Quantum Yield Measurements....................................................................... 3

Figure S1. SEM and Edge Length Distribution of AgNP..................................................... 4

Figure S2. Absorption and Emission Spectra of 1................................................................... 5



Single Molecule Fluorescence Microscopy Experimental Details..........................................S6

Single Molecule Data Analysis Protocol..............................................................................

Figure S4. Representative Single Molecule Fluorescence Bursting without AgNP....................S7

Figure S5. Representative Single Molecule Fluorescence Bursting with AgNP........................S8

Figure S6. Diagram of TIRFM experimental design............................................................... 9

Figure S7. Emission spectra of films of $\mathbf{1}$ or $\mathbf{2}$ in the presence and absence of AgNP..............S10

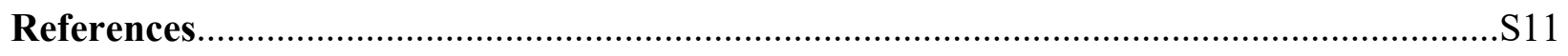




\section{Materials and Instrumentation}

Reagents and solvents were purchased from Sigma-Aldrich, Fisher Scientific and ACP Chemicals, and used as received. Ultrapure deionized water (Milli $\Omega, 18.2 \mathrm{M} \Omega$ ) was obtained from a Millipore Purification System. Analytical thin-layer chromatography was performed using aluminum sheets coated with silica $\left(60, \mathrm{~F}_{254}\right)$. NMR spectra were recorded at room temperature with a Bruker Avance $400 \mathrm{MHz}$ spectrometer. Chemical shifts $(\delta)$ are reported in parts per million (ppm), relative to residual $\mathrm{CDCl}_{3}$ solvent signal at $7.26 \mathrm{ppm}$. Scanning electron microscopy was performed using a Hitachi S-5200 at $5 \mathrm{kV}$ acceleration voltage. Samples were drop casted onto carbon coated square mesh copper grids (Electron Microscopy Sciences, CF400-CU-UL) and dried under ambient conditions. Steady-state absorption spectra were recorded with an Agilent Cary $60 \mathrm{UV}$-visible spectrophotometer using quartz cells with a path length of $1 \mathrm{~cm}$. Steady-state emission spectra were recorded with an Agilent Cary Eclipse spectrofluorometer. Thin polymer films were prepared by spin coating $150 \mu \mathrm{L}$ of a $500 \mu \mathrm{M}, 20 \%$ (w/v) polyvinylpyrrolidone (PVP, $55 \mathrm{kDa}$ ) methanolic solution of $\mathbf{1}$ or $\mathbf{2}$ onto glass slides or coverslips (Fisher Scientific) at $1000 \mathrm{rpm}$ for $40 \mathrm{~s}$. Brownian motion was partially restricted by the increased viscosity of the polymer film but any fluorophore motion was still random and without AgNP specificity.

\section{Synthesis}

BODIPY 1 was synthesized according to our reported protocol, ${ }^{\mathrm{S} 1}$ and characterization by thinlayer chromatography, mass spectrometry, ${ }^{1} \mathrm{H}$ and ${ }^{13} \mathrm{C}\left\{{ }^{1} \mathrm{H}\right\}$ NMR spectroscopy were in excellent agreement with our previously reported values. ${ }^{1} \mathrm{H} \mathrm{NMR}\left(400 \mathrm{MHz}, \mathrm{CDCl}_{3}\right): \delta 7.72(2 \mathrm{H}, \mathrm{s})$, 7.52-7.62 (5H, m), $6.98(2 \mathrm{H}, \mathrm{dd}, \mathrm{J}=1.1,4.2 \mathrm{~Hz}), 6.87-6.89(2 \mathrm{H}, \mathrm{m}), 6.80-6.82(2 \mathrm{H}, \mathrm{m}), 6.50$ $(2 \mathrm{H}, \mathrm{dd}, \mathrm{J}=2.0,4.3 \mathrm{~Hz}) .{ }^{13} \mathrm{C}\left\{{ }^{1} \mathrm{H}\right\} \mathrm{NMR}\left(\mathrm{CDCl}_{3}, 400 \mathrm{MHz}\right): \delta 150.9,147.3,145.1,134.9$, $133.8,132.2,130.8,130.5,128.5,119.5,118.9,109.8 .{ }^{11} \mathrm{~B}\left\{{ }^{1} \mathrm{H}\right\} \mathrm{NMR}\left(\mathrm{CDCl}_{3}, 400 \mathrm{MHz}\right): \delta 6.6$ (s). EI-MS [M] $]^{+}=338.05 \mathrm{~m} / \mathrm{z}$.

BODIPY 2 was prepared by adding 1.5 equiv $(0.057 \mathrm{mmol}) \mathrm{HCl}$ to 1 equiv $(0.038 \mathrm{mmol})$ of 1 dissolved in $20 \mathrm{~mL}$ of methanol, and stirring for approximately 5 minutes. The reaction was monitored by analytical TLC, and conversion was apparent by the immediate appearance of strong fluorescence emission upon addition of $\mathrm{HCl}$, visualized using a TLC lamp. Purification by preparative TLC (95:5 DCM:MeOH) afforded 2 as an orange solid, in 62\% yield. ${ }^{1} \mathrm{H}$ NMR (400 $\left.\mathrm{MHz} \mathrm{CDCl}_{3}\right): \delta 7.92(2 \mathrm{H}, \mathrm{s}), 7.52-7.60(5 \mathrm{H}, \mathrm{m}), 6.92(2 \mathrm{H}, \mathrm{d}, \mathrm{J}=3.7 \mathrm{~Hz}), 6.54-6.55(2 \mathrm{H}, \mathrm{m})$, $3.10(6 \mathrm{H}, \mathrm{s})$.

AgNP were prepared according to our established photochemical protocol reported in detail elsewhere. ${ }^{\mathrm{S} 1}$ Characterization of the nanomaterial was in accordance with previous batches. 


\section{Fluorescence Quantum Yield Measurements}

Fluorescence quantum yields of $\mathbf{1}$ and $\mathbf{2}$ in methanol were determined using fluorescein in $0.1 \mathrm{M}$ $\mathrm{NaOH}\left(\Phi_{R}=0.95\right)^{\mathrm{S} 2}$ as the standard (denoted by subscript $R$ below). Excitation wavelength was $460 \mathrm{~nm}$, and emission spectra were integrated from $475-800 \mathrm{~nm}$. Quantum yield of fluorescence was then computed according to the following equation:

$$
\Phi_{f l}=\Phi_{R} \frac{I}{I_{R}} \frac{A_{R}}{A}\left(\frac{\eta}{\eta_{R}}\right)^{2}
$$

where $I, A$ and $\eta$ represent integrated fluorescence intensity, absorption (below 0.1) at the excitation wavelength, and refractive index of the solvent, respectively. 



Figure S1. SEM image and size distribution histogram for AgNP. SEM image recorded at $\times 40,000$ magnification and acceleration voltage of $5 \mathrm{kV}$. Scale bar $=200 \mathrm{~nm}$. 




Figure S2. Absorption and emission $\left(6.9 \mu \mathrm{M}, \lambda_{\mathrm{Ex}}=450 \mathrm{~nm}, \mathrm{MeOH}, 20{ }^{\circ} \mathrm{C}\right)$ spectra of $\mathbf{1}$. Excitation slit width $=5 \mathrm{~nm}$, emission slit width $=10 \mathrm{~nm}$, PMT voltage $=625 \mathrm{~V}$.

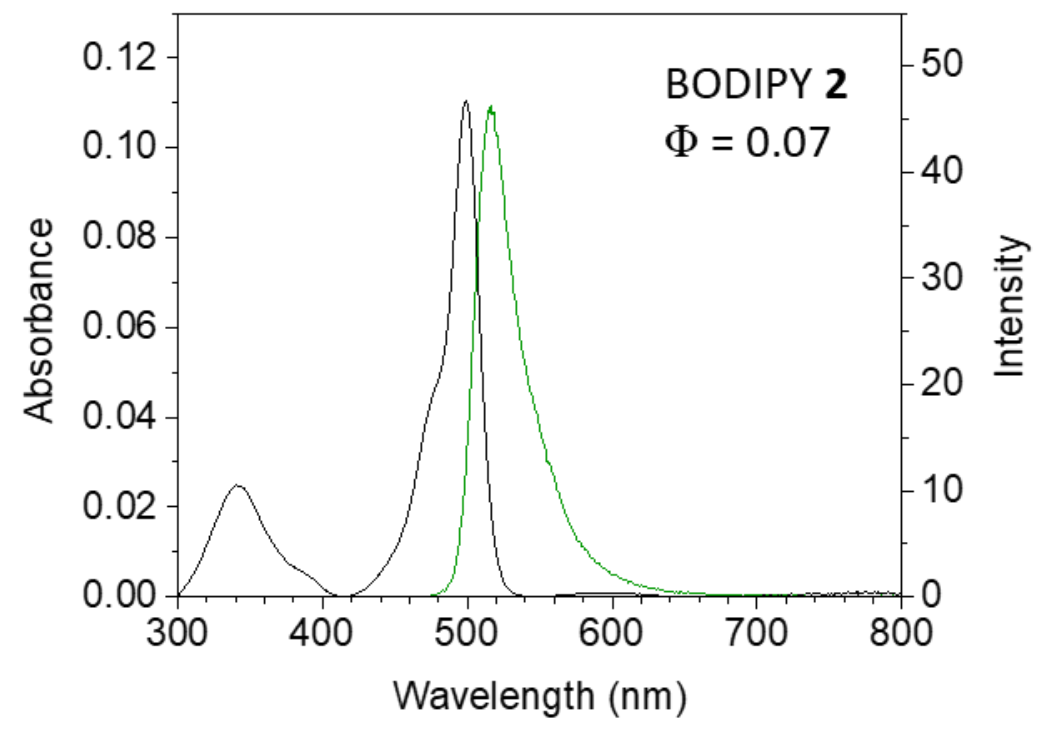

Figure S3. Absorption and emission $\left(3.2 \mu \mathrm{M}, \lambda_{\mathrm{Ex}}=460 \mathrm{~nm}, \mathrm{MeOH}, 20^{\circ} \mathrm{C}\right)$ spectra of 2. Excitation slit width $=5 \mathrm{~nm}$, emission slit width $=5 \mathrm{~nm}$, PMT voltage $=625 \mathrm{~V}$. 


\section{Single Molecule Fluorescence Microscopy Experimental Details}

Total Internal Reflection Fluorescence Microscopy was performed using an Andor Diskovery multi-modal imaging system by Quorum Technologies Inc., coupled to a Leica DMi8 inverted microscope. A $63 \times$ oil immersion objective (NA 1.49) and 1.8 $\times$ camera relay provided a total of 108× magnification. A $488 \mathrm{~nm}$ direct modulation laser source from an Andor Integrated Laser Engine operating at $4.2 \mathrm{~mW}$ provided sample excitation. Fluorescence emission and excitation light reflected back from the sample passed through a $50 \mathrm{~nm}$ band pass emission filter centred at $525 \mathrm{~nm}$ before being collected by an Andor iXon 897 EMCCD camera. The EM Gain was set to 100 and exposure time was $50 \mathrm{~ms}$ (i.e. image sequences were recorded at $20 \mathrm{frames} / \mathrm{s}$ ). Quorum Wave FX acquisition software (powered by MetaMorph) was used to control instrument parameters and to obtain images consisting of $512 \times 512$ pixels (px) at a scale of $153 \mathrm{~nm} / \mathrm{pixel}$, for a total image area of $78.14 \mu \mathrm{m} \times 78.14 \mu \mathrm{m}\left(6105.86 \mu \mathrm{m}^{2}\right)$. Samples for single molecule fluorescence microscopy were prepared by spin coating a $100 \mathrm{nM}$ solution of $\mathbf{1}$ or $\mathbf{2}$ dissolved in


Scientific) at $1000 \mathrm{rpm}$ for $40 \mathrm{~s}$. In some cases, coverslips had previously been functionalized with a layer of AgNPs by drop casting and drying under ambient conditions. Previous research has experimentally verified that this protocol produces polymer films $70 \pm 12 \mathrm{~nm}$ thick, which represents the maximum possible distance between $\mathrm{AgNP}$ and $\mathbf{1}$ or $2 .{ }^{\mathrm{S} 1} \mathrm{In}$ all cases, samples were transported in the dark and used immediately following preparation.

\section{Single Molecule Data Analysis Protocol}

Image analysis was performed using Fiji, MatLab, OriginPro and Microsoft Excel software, based on modified literature protocols. ${ }^{\text {S1 }}$ In brief, raw TIRF image sequences were imported into Fiji, where background subtraction was performed using a 10 px rolling ball algorithm. Videos were then analysed using an in-house written MatLab protocol which builds upon the established LocalizerMatlab.mex64 script developed by Peter Dedecker (University of Luven, Belgium) ${ }^{\mathrm{S} 3}$ for the localization of single molecule fluorescence bursting in super-resolution microscopy. Essentially, our algorithm conducts a frame by frame analysis of TIRF image sequences using a Generalized Likelihood Ratio Test (GLRT) to categorize relative pixel intensity fluctuations and fit them to a 2D Gaussian approximation of the Point Spread Function describing fluorophore emission. The algorithm then filters the results to remove duplication and returns the xy coordinates of potential emitters. These locations were used as the centre points for circular, 1.5 px radius regions of interest (ROIs), automatically imported into the Fiji ROI manager tool using an in-house written macro. From here, Fiji was used to measure the mean intensity within each ROI for each frame individually, across 1000 frames. These data were utilized to plot intensitytime trajectories and perform statistical analysis of fluorescence burst intensities using OriginPro and MS Excel. Burst intensities for individual intensity-time trajectories were taken as the difference between peak intensity and baseline signal. Mean fluorescence bursting intensity $(\mu)$ was calculated according to the following equation for the mean of a log-normal distribution:

$$
\mu=e^{\left[\ln (x c)+0.5 w^{2}\right]}
$$

where $x c$ (center) and $w$ (scaling factor) were computed by OriginPro software. Similarly, standard deviation $(\sigma)$ of a log-normal distribution was given by the following equation:

$$
\sigma=e^{\left[\ln (x c)+0.5 w^{2}\right]}\left[e^{w^{2}}-1\right]^{1 / 2}
$$





Figure S4. Example single molecule fluorescence bursting trajectories for TIRFM image sequences recorded in the absence of AgNP. 



Figure S5. Example single molecule fluorescence bursting trajectories for TIRFM image sequences recorded in the presence of AgNP. The top two traces (A-B) illustrate high-intensity plasmophoric MEF bursting and the bottom two traces (C-D) demonstrate bursting due to increased fluorophore excitation. 


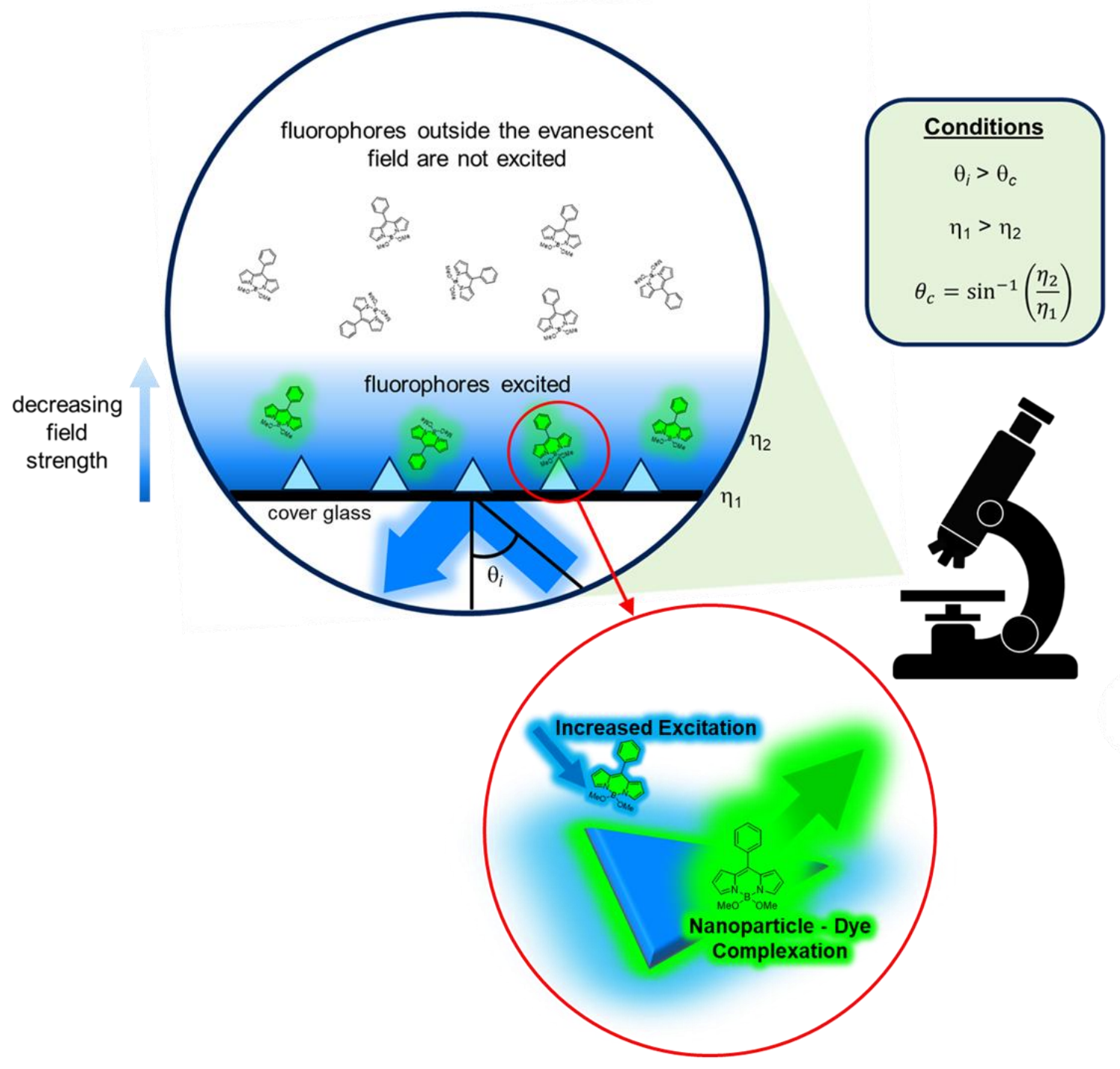

Figure S6. Diagram of TIRFM experimental design. 

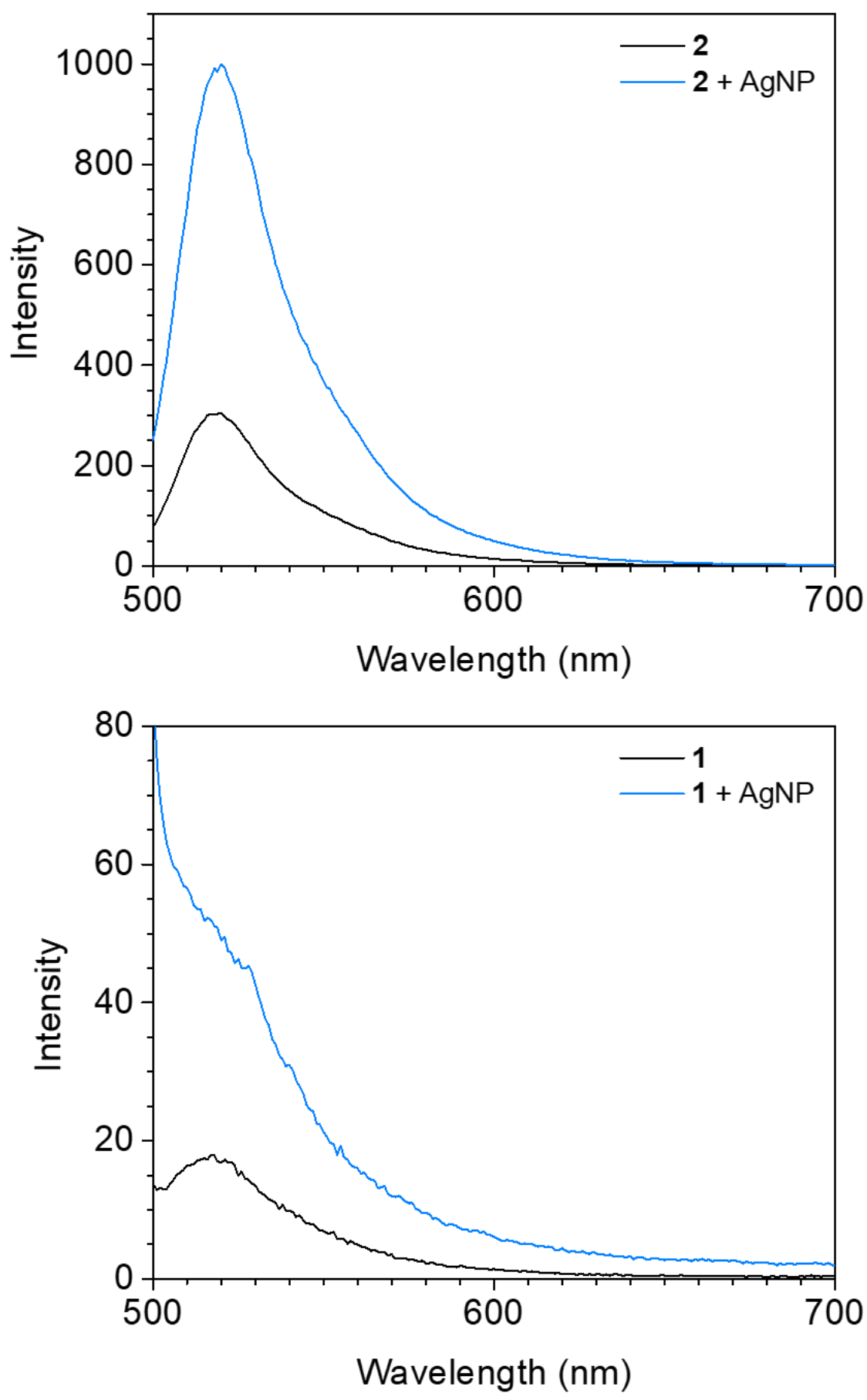

Figure S7. Emission spectra of PVP films of $\mathbf{1}$ or $\mathbf{2}$ in the presence and absence of $\mathrm{AgNP}\left(20^{\circ} \mathrm{C}\right.$, $\left.\lambda_{\mathrm{Ex}}=490 \mathrm{~nm}\right)$. 


\section{References}

S1 Dogantzis, N. P.; Hodgson, G. K. Impellizzeri, S. Nanoscale Adv. 2020, 2, 1956.

S2 Lakowicz, J. R. Principles of Fluorescence Spectroscopy, $3^{\text {rd }}$ edition; Springer: New York, 2006.

S3 Dedecker, P.; Duwe, S.; Neely, R. K.; Zhang, J. J. Biomed. Opt. 2012, 17, 126008. 